\title{
Maintaining cost-effective access to antiretroviral drug therapy through a collaborative approach to drug procurement, consensus treatment guidelines and regular audit: the experience of London HIV commissioners and providers
}

\author{
Claire Foreman, ${ }^{1}$ Brian Gazzard, ${ }^{2}$ Margaret Johnson, ${ }^{3}$ Peter Sharott, ${ }^{1}$ \\ Simon Collins ${ }^{4}$
}

${ }^{1}$ London Specialised

Commissioning Group, London, UK

${ }^{2}$ HIV/GU Unit, Chelsea and Westminster Hospital NHS Foundation Trust, London, UK ${ }^{3}$ Ian Charleson Day Centre, Royal Free Hampstead NHS

Trust, London, UK

${ }^{4}$ HIV i-Base, London, UK

\section{Correspondence to} Claire Foreman, Senior Commissioning Manager, London Specialised Commissioning Group, Portland House, Stag Place, London SW1E 5RS, UK; claire. foreman@londonscg.nhs.uk

Accepted 4 January 2012

\section{ABS}

ABSTRACT

Background In the UK, meeting the $£ 20$ billion efficiency challenge in the NHS requires new approaches to protect quality and improve productivity. In London, clinicians, people living with HIV and commissioners are collaborating to reduce the cost of antiretrovirals as part of the Quality Innovation Productivity and Prevention agenda.

Objectives To describe how collaboration in antiretroviral procurement in 2011/2012 aimed to significantly reduce drug acquisition costs, ensure equity of prescribing and protect the quality and experience of care and treatment for patients.

Methods Greater clinical leadership and engagement and involvement of patient representatives enabled an approach to drug procurement focused on clinical outcomes at a patient and population level while reducing cost. Consensus guidelines for implementation were developed and agreed by all London lead clinicians while people living with HIV produced a patient information leaflet to explain the tender process and outcomes. A planned audit is underway at all services to monitor prescribing changes and outcomes for those on treatment.

Results HIV clinicians, pharmacists and patient representatives were directly involved in this novel therapeutic tendering approach to antiretroviral drug procurement. Modelling indicates that $£ 8-£ 10$ million savings will be released through the process over 2 years.

Conclusions Clinically led therapeutic tendering of antiretroviral drugs provides an opportunity to protect quality and improve productivity in HIV. The approach is novel in HIV in the UK, and the emergent learning has implications for quality and cost improvement in HIV spending in the UK and potentially in other countries.

\section{INTRODUCTION}

\section{Commissioning health services in the UK}

In the UK, Primary Care Trusts (PCTs) are the statutory bodies with responsibility for commissioning health services for their patient populations. Specialised services are defined in law as those services with a planning population of $>1$ million people and typically include services delivered by fewer than 50 providers in England. ${ }^{1}$
Currently, the Department of Health makes recurrent funding allocations directly to PCTs on the basis of the relative needs of their populations and in line with relevant policy. A weighted capitation formula determines each PCT's target share of available resources to enable them to commission similar levels of health services for populations in similar need and to reduce avoidable health inequalities. In turn, PCTs work with Specialised Commissioning Groups to agree annual budgets for the commissioning of specialised services on their behalf.

As a result of the wider economic climate and rising costs of care, NHS budgets are under unprecedented financial pressure. The Operating Framework for the $\mathrm{NHS}^{2}$ sets out the requirement to deliver up to $£ 20$ billion of efficiency savings by 2014/2015. In response to this challenge, the NHS is developing Quality Innovation Productivity and Prevention plans which aim to improve quality and cost through innovation and effective prevention strategies.

\section{The challenge of commissioning for HIV care and treatment in the UK}

The epidemiology of HIV poses significant commissioning challenges in the context of financial constraint. According to the Health Protection Agency, an estimated 91500 people were living with HIV in the UK in 2010, of whom 69400 were in care and treatment. By the end of 2012, the number of people living with HIV infection (diagnosed and undiagnosed) in the UK was predicted to have reached 100000 . In addition to increases in the number of people living with HIV, commissioning intentions need to take account of the increasing proportion of patients taking antiretrovirals. HIV surveillance data indicate that in 2010, around half of those diagnosed as having HIV infections in the UK had a CD4 count of $<350$, below the recommended threshold for beginning HIV treatment. ${ }^{3}$ Current debate about the role of treatment as prevention is likely to increase the proportion of people on treatment further

Additional commissioning challenges included lack of access to patient identifiable data, planning for open access to HIV services and ensuring equity in the context of highly individualised treatments, including the use of off-licence treatment. 


\section{The London experience of commissioning for HIV care and treatment}

In London, HIV care and treatment is collaboratively commissioned by the London Specialised Commissioning Group on behalf of 31 PCTs. An engagement structure with clinically led subgroups for Drugs and Treatment and Audit and Outcomes bring together commissioners, clinicians and patient representatives to plan, deliver and evaluate HIV care and treatment services. Twenty-three HIV-specialised service providers are commissioned to deliver care and treatment services on an open access basis.

Commissioning for improvements in quality and cost has been underway for a number of years. London HIV care and treatment contracts include four clinical outcome measures related to access to care, effectiveness of treatment, effectiveness of care and mortality. Outcomes are monitored for each provider using Health Protection Agency surveillance data. Commissioning for clinical outcomes in this way is novel and probably unique, and the standard of clinical care achieved is internationally renowned. By 2010, the quality of HIV care received in London was high: $80 \%$ of newly diagnosed patients were seen in a HIV clinic within 1 month of diagnosis, 90\% had an undetectable viral load ( $<50$ copies $/ \mathrm{ml}$ ) 1 year after starting therapy and $93 \%$ of those in care for more than a year had a CD 4 count above 200 cells $/ \mathrm{mm}^{3}$.

In 2011/2012, the budget available to commission HIV care and treatment in London for approximately 30000 people was the same as the previous year at around $£ 250$ million. The number of people living with HIV and accessing care and treatment in London has almost doubled in the last decade and increases annually by approximately 1800 new patients. By $2010,83 \%$ of those in care were on antiretroviral treatment. ${ }^{5}$

\section{A new approach to antiretroviral drug procurement: therapeutic tendering}

London NHS Trusts spend around approximately $£ 170$ million per annum on antiretroviral drugs, representing almost $20 \%$ of their total drugs expenditure. Antiretroviral procurement on a collaborative pan-London basis has successfully secured equitable access to therapy for patients at the best-negotiated prices for a number of years. With base case funding, commissioners asked the clinically led Drugs and Treatment Sub Group to consider opportunities to secure further reductions in drug acquisition costs.

\section{METHODS}

In October 2010, the London HIV Drugs and Treatment Sub Group agreed to include a therapeutic tender approach in the antiretroviral procurement process for 2011/2012. The aim of the therapeutic tender was to identify the potential for agreeing first-line drug choices which would stimulate volume growth and significantly reduce acquisition costs while protecting clinical care and outcomes for all HIV-infected individuals. Outcomes of the tender process would need to demonstrate evidence-based use of antiretroviral drugs, in line with current treatment guidelines, and ensure continued performance on the London clinical outcome measures. In addition, the process would need to support the primacy of the clinical consultation between HIV doctor and patient. The freedom of the individual clinician to prescribe the most appropriate drug for the patient and full involvement of the person living with HIV in treatment decision-making processes were confirmed as fundamental principles. On this basis, the clinicians and patient representatives involved agreed that using least expensive clinically appropriate alternatives is reasonable in order to balance the needs of individuals and populations in a resource-constrained environment.

The Drugs and Treatment Sub Group delegated a multidisciplinary panel involving doctors, commissioners, specialist pharmacy, public health and patient representatives to take forward the process. Meetings were held with pharmaceutical companies to explain the process, and invitations to tender were placed in the Official Journal of the European Union. Pharmaceutical companies were invited to tender based on both continuation of existing prescribing volumes and on a cost and volume matrix. The procurement process was supported by the Department of Health's Commercial Medicines Unit.

In December 2010, tenders were reviewed by the panel and recommendations for accepting tenders were referred to all clinical leads for discussion and approval. Consensus prescribing messages were developed and agreed by lead HIV clinicians for clinically appropriate use of the least expensive nucleoside analogue reverse transcriptase inhibitors and protease inhibitors (PIs).

\section{RESULTS}

\section{Clinical considerations in therapeutic tendering}

HIV clinicians, commissioners and patient representatives reviewed the submitted tenders in the context of evidence and existing treatment guidelines, taking account of toxicity, tolerability and convenience. Following the review of the tenders, there were two areas where potential changes in treatment were agreed.

Consideration would be given first to the use of Kivexa ${ }^{\circledR}$ (abacavir and lamivudine fixed-dose combination) before tenofovir in the fixed-dose combinations with emtricitabine (Truvada ${ }^{\circledR}$ ) or with emtricitabine and efavirenz (Atripla ${ }^{\circledR}$ ) or other Nucleoside analogue Reverse Transcriptase Inhibitor backbone combinations in patients starting antiretroviral therapy for the first time or where a switch of treatment was being considered. As in the current routine clinical practice, a negative HLA-B*5701 test would be required. Additional exclusion criteria for using abacavir would include high baseline viral load (>100000 copies) because of the results of the ACTG5202 clinical trial. A high Framingham risk score for cardiovascular disease ( $>10 \%$ over 10 years) would also constitute an exclusion due to potential concerns about possible cardiovascular risk with abacavir. A recent ACTG review found no signal of enhanced cardiovascular events in individuals with a low cardiovascular risk in randomised clinical trials. The advice also made clear that there is a range of pre-existing abnormalities in addition to cardiovascular risk where one nucleoside analogue backbone might be preferred over another. While there is no definitive data that tenofovir should not be used in individuals with a high risk of development of renal insufficiency, abacavir is often the preferred option. These recommendations are for people starting treatment. The outcome of the tender did not include a financial incentive to change nucleoside treatment for patients currently on stable treatment.

Second, with regard to PIs, a number of head-to-head studies have demonstrated that many of the routinely used drugs have very similar efficacy profiles but can have different toxicity profiles. Therefore, when choosing a PI for use in first PI therapy, it is again reasonable to take cost into account. As a result of the tendering process, atazanavir/ritonavir offered the opportunity for considerable cost saving if considered first compared with using other once-daily and twice-daily PIs. 
With PIs, the tender outcome included a financial incentive if some patients currently on PI-based treatment were to switch to atazanavir/ritonavir. Therapy would be reviewed for switching treatment but it was agreed that this would only be considered when clinically appropriate and with the patient's consent.

The most recent edition of the UK (BHIVA) treatment guidelines was published in 2008 and therefore does not capture all current clinical practice. Clinicians involved in the process discussed existing evidence in relation to clinical efficacy and adherence in agreeing the outcome of the tender and the guidelines to support implementation. As $>100$ physicians are involved across London in initiating or changing antiretroviral treatment for HIV patients, the clinical guidelines were developed to ensure consistent approaches across all services. These were widely disseminated through HIV services, the Patient and Public Engagement Sub Group and via community information websites including Aidsmap and HIV i-Base. ${ }^{6}$

Patient information to support the process was been crucial. The HIV Patient and Public Engagement Sub Group developed a leaflet explaining the context for the tender and the implications of the outcomes on patient care. Leaflets have been disseminated via clinics and advocacy organisations. ${ }^{7}$

\section{Implementation and audit}

Modelling suggests that the agreed contracts-which cover a 2-year period from April 2011-will deliver £8-£10 million of savings if the volumes and prices for the antiretroviral drugs are achieved for London as a whole. Monitoring of drug usage is undertaken on a monthly basis and reported to clinicians at each provider as well as being reviewed by the Drugs and Treatment Sub Group.

Of paramount importance to commissioners, clinicians and patients is ensuring that clinical quality of care is maintained and clinical outcomes protected. Equity in implementation of the clinical guidelines is also critical to success.

Providing assurance that guidelines are being implemented and clinical outcomes protected requires a programme of audit. All services in London are participating in an audit to track all patients affected by the changes to monitor implementation and the clinical outcomes. The clinically led Audit and Outcomes Sub Group will be reviewing the audit findings over the next 2 years.

\section{DISCUSSION AND CONCLUSIONS}

The challenge for every healthcare system is how to pay for the care it wishes to provide. The success of HIV care and treatment means more people are living longer and healthier lives. The continued success will depend on these and more people having continued access to effective therapy, which must be paid for. Everyone involved-from commissioners, clinical practitioners, public health specialists, patients and their representativesagree on the importance of securing access to effective therapy for the future. Finding solutions to this challenge must be a collaborative process. This year's work on extending the approach to procurement of antiretrovirals is proving successful in this regard. Modelling suggests that the work is on target to deliver the savings required and engagement of clinicians and patients in procurement has been significantly enhanced.

One of the important tenets of medical ethics requires consideration of the costs of drugs prescribed and the potential impact of prescribing in a cost-constrained system. Suppressive HIV treatment-whatever the drug used-remains a cost-effective way of saving years of good quality life and compares favourably with the treatment for many other chronic diseases. Where drugs have equivalent efficacy and toxicity, prescribing the least expensive alternative can deliver individual and public health benefit for those living with HIV.

This work has highlighted consensus on the principle that cost is a reasonable consideration for individual clinicians and patients to take into account when selecting treatment options of similar efficacy. Concerns have been expressed about how the changes may affect the doctor-patient relationship. It is essential that there is always a full discussion of treatment options including efficacy, risks and cost rationale with patients, and this has been emphasised throughout London HIV services. There is a strong tradition of patient engagement and advocacy in HIV care in London and the UK which means patients, doctors and commissioners have a history of working together to protect quality while challenging and addressing inefficiencies. In many London services, patients and treatment advocates have led the way in developing or supporting improvements such as increased peer support, more flexible clinic arrangements and home delivery of medicines.

The tender resulted in guidelines for use of preferred antiretrovirals that would deliver considerable cost reduction without negatively impacting on the likely efficacy or tolerability of treatment, as long as the guidelines were followed. For many patients, the newer guidelines represented potential improvements over their previous care, often with reduced pill burden. The outcome limited the impact on patients who were already stable on treatment and ensured access to the highest quality care with minimal disruption to patients. In future, it is possible that such processes could result in tenders where less potent antiretroviral drugs were substantially less expensive or where savings could only be realised through more widespread switching of patients currently on stable treatment. Both of these issues need to be considered as part of future procurement processes in order that quality of treatment continues to be optimised, disruption to patients is minimised and drug acquisition costs continue to improve.

For London, an important question is what will happen at the end of this tender period. Some antiretroviral drugs have patents due to expire. There will be new data on efficacy and toxicity of existing antiretroviral drugs and new drugs will be licensed. The commissioning landscape too is in a process of change which will impact on the way in which procurement exercises are likely to be undertaken. An ongoing process of horizon scanning will be required to inform future recommendations for drug procurement and to continue to develop creative solutions to manage increased demand within existing resources. While we continue to advocate for more resources for HIV prevention, care and treatment, we need to continue to identify opportunities for cost improvement, so that freed up resources can be reinvested in HIV services, and it can be shown that the current excellent clinical care of HIV is being maintained despite financial constraints.

\section{Learning points}

A major challenge to the tendering process was how to engage with all stakeholders. The commercially confidential nature of tendering restricts opportunities for consultation or the sharing of specific details during the procurement process in order that the outcome is not prejudiced or subject to legal challenge. Despite the engagement achieved in the HIV therapeutic tender through the use of clinical leads and patient representatives, the announcement of the completed process raised some concerns 
from community advocates who were not directly involved in the process.

As well as ensuring procurements continue to be Official Journal of the European Union compliant, future collaborative tendering will need to do more to communicate and reassure stakeholders of the robustness and transparency of commercially confidential processes. For example, there would be benefit in greater discussion prior to procurements on the key objectives and acceptability of potential outcomes. Approaches will need to explore how to achieve greater involvement in the preparation phase, how to share non-confidential information with key groups and how outcomes can be widely publicised. Delivering broader and deeper engagement in the context of procurement rules requires clear structures for involvement and for decision making, and these will need to evolve further.

In summary, reflections on the process so far indicate a number of learning points, summarised in the box below.

Although the learning and outcomes for London are emergent, indications are that collaborative approaches can secure

\section{Key messages}

- Delivering effective solutions to reducing cost and protecting quality requires extensive and ongoing clinical engagement.

- Involvement of people living with HIV and their advocates in Quality Innovation Productivity and Prevention plan and procurement exercises is fundamental.

- Novel approaches in procurement can reduce drug acquisition costs, and similar approaches could be applied to other aspects of care and therapy.

- The London experience indicates that quality can be protected while costs are reduced. Treatment remains in line with guidelines, and disruption to patients has been minimised.

- Processes and outcomes need to be widely communicated and learning used to improve future processes. equitable prescribing practice, quality clinical outcomes and cost reductions. This depends on a managed process that can achieve clinical leadership and extensive clinical and patient engagement in a commercially confidential context. Learning from and building on these processes will be critical if HIV is able to meet the Quality Innovation Productivity and Prevention challenge over the coming years.

Acknowledgements Thanks to all contributors, to all London HIV Clinical Leads, HIV PPE Sub Group and London HIV Pharmacists.

\section{Competing interests None.}

Patient consent Obtained.

Contributors CF is the Lead Commissioner for HIV and leads on ensuring clinical and patient engagement in planning. CF is the lead author for this article. BG is the Chair of the Drugs \& Treatment Sub Group, which led the procurement process and produced the consensus guidelines. BG has co-authored this article. MJ is the Vice Chair of the Drugs \& Treatment Sub Group. MJ has co-authored this article. PS leads the negotiations on antiretrovirals on behalf of London. PS has co-authored this article. SC acted as one of the treatment advocate representatives in the procurement. SC has co-authored this article. This article sets out the experience of London commissioners, providers and patients in therapeutic tender of ARVS.

Provenance and peer review Commissioned; externally peer reviewed.

\section{REFERENCES}

1. NHS. Specialised Services National Definitions Set. http://www.specialisedservices. nhs.uk/info/specialised-services-national-definitions (accessed 28 Dec 2011).

2. Department of Health. The operating framework for the NHS in England 2012/13. http://www.dh.gov.uk/prod_consum_dh/groups/dh_digitalassets/documents/ digitalasset/dh 131428.pdf (accessed 28 Dec 2011).

3. Health Protection Agency. HIV in the United Kingdom: 2011 Report. http://www. hpa.org.uk/webc/HPAwebFile/HPAweb_C/1317131685847 (accessed 28 Dec 2011).

4. Health Protection Agency. HIV in the United Kingdom: 2010 Report. Health Protection. 1, Slide 1. http://www.hpa.org.uk/webc/HPAwebFile/HPAweb_C/ 1287145421738\#277 (accessed 28 Dec 2011).

5. Health Protection Agency. HIV-diagnosed persons seen for HIV care. http://www hpa.org.uk/webc/HPAwebFile/HPAweb_C/1221482343523 (accessed 28 Dec 2011).

6. AIDSMAP. HIV Drug Prescribing in London to Change From this Month. http://www. aidsmap.com/HIV-drug-prescribing-in-London-to-change-from-this-month/page/ 1753495/ (accessed 28 Dec 2011).

7. London Specialised Commissioning Group. Changes to How Your HIV Medication is Prescribed. http://www.londonspecialisedcommissioning.nhs.uk/documents/712.pdf (accessed 28 Dec 2011). 